\title{
Hydrothermal instability of thermocapillary convection in large-Prandtl-number liquid bridges under microgravity
}

\author{
Qi-Sheng Chen *, Ya-Chao Liu \\ National Microgravity Laboratory, Institute of Mechanics, Chinese Academy of Sciences, 15 Bei-Si-Huan-Xi Road, Beijing 100080, China
}

Received 3 November 2006; received in revised form 29 August 2007; accepted 29 August 2007

\begin{abstract}
Linear stability analysis was performed to study the mechanism of transition of thermocapillary convection in liquid bridges with liquid volume ratios ranging from 0.4 to 1.2 , aspect ratio of 0.75 and Prandtl number of 100 . 2-D governing equations were solved to obtain the steady axi-symmetric basic flow and temperature distributions. 3-D perturbation equations were discretized at the collocation grid points using the Chebyshev-collocation method. Eigenvalues and eigenfunctions were obtained by using the Q-R method.

The predicted critical Marangoni numbers and critical frequencies were compared with data from space experiments. The disturbance of the temperature distribution on the free surface causes the onset of oscillatory convection. It is shown that the origin of instability is related to the hydrothermal origin for convections in large-Prandtl-number liquid bridges.
\end{abstract}

(C) 2007 COSPAR. Published by Elsevier Ltd. All rights reserved.

Keywords: Microgravity; Instability; Liquid bridge

\section{Introduction}

Instability of thermocapillary convection can be caused by different mechanisms such as hydrothermal, hydrodynamic, hydrothermal wave, etc. The hydrothermal instability explains that the onset is caused by the temperature disturbance especially on the free surface. The hydrodynamic instability explains that the onset is caused by the velocity disturbance especially when the Reynolds number is high. The hydrothermal wave instability considers that the transition is caused by waves on the free surface when the free surface is deformable. Researchers have performed experiments and linear stability analysis to investigate the effects of the liquid volume on the onset of the oscillatory convection in liquid bridges.

Cao et al. (1992) measured the critical temperature differences in liquid bridges of $10 \mathrm{cSt}$ silicone oil contained by rods of $2 \mathrm{~mm}$ in diameter by an interference method in

\footnotetext{
* Corresponding author. Tel.: +8610 82544092.

E-mail address: qschen@imech.ac.cn (Q.-S. Chen).
}

which a light sheet is used to pass the liquid bridge with a curved free surface and produce an interference pattern. They found that the curve of the critical temperature difference versus liquid volume has two branches, corresponding to slender and fat bridges, respectively. Further studies of features of the critical temperature difference versus liquid volume in liquid bridges confined by rods of $3 \mathrm{~mm}$ in diameter were conducted ( $\mathrm{Hu}$ et al., 1994). It was found that there is a gap region in the curves of critical temperature difference versus liquid volume, in which the convection remains steady when increasing the temperature difference until the bridge is broken. In their experiments, maximum of $100 \mathrm{~K}$ temperature difference could be obtain which corresponds to $M a=10,164$. Yao et al. (1997) conducted microgravity experiment in liquid bridges confined by rods of 3 and $4 \mathrm{~mm}$ diameters using the 10-s drop shaft facility. Several cases with different liquid volumes were studied which show that the critical temperature differences are different in 1-g and microgravity conditions. Albanese et al. (1995) performed onset experiment during the D2 spacelab mission on the Advanced Fluid Physics Module (AFPM). A liquid with a relatively high Prandtl number 
$(P r=74)$ was used ( $5 \mathrm{cSt}$ silicone oil). The critical Marangoni number is dependent on the disk diameter. The critical Marangoni numbers based on the disk radius are $7.6 \times 10^{4}$ and $1.10 \times 10^{5}$ for aspect ratio of 0.75 and disk diameters of $45 \mathrm{~mm}$ and $60 \mathrm{~mm}$, respectively. Because of the gravitational effects, the values of the critical Marangoni numbers measured in the ground-base experiments are different from these measured in space experiments for the same Prandtl number and aspect ratio.

Chen and $\mathrm{Hu}$ (1997) calculated the flow and temperature patterns in a floating half zone using the Chebyshevcollocation method. They further obtained the curves of the critical Marangoni number versus liquid volume in liquid bridges of $\mathrm{Pr}=50$ in microgravity conditions (Chen and $\mathrm{Hu}, 1998)$. The critical curves consist of two branches corresponding to slender and fat bridges, respectively. Shevtsova and Legros (1998) obtained the stability boundary for $\operatorname{Pr}=105, A=0.67$ and a wide range of liquid volumes. Sumner et al. (2001) obtained the critical curve of Marangoni number versus liquid volume for $5 \mathrm{cSt}$ silicone oil and $A=0.75$ using the energy-stability analysis. Nienhüser and Kuhlmann (2002) investigated the stability of thermocapillary flows in non-cylindrical liquid bridges. The perturbation equations were discretized by the second-order finite difference method.

In this paper, we calculate the critical Marangoni numbers and critical frequencies for $\operatorname{Pr}=100, A=0.75$ and different liquid volumes, and analyzed the mechanism of instability of the thermocapillary convections.

\section{Physical and mathematical model}

A liquid bridge is formed by inserting a liquid such as silicone oil in between two rods of diameter $D$, which are separated by a distance $L$ (Fig. 1). Thermocapillary convection will onset when the two rods are applied with different

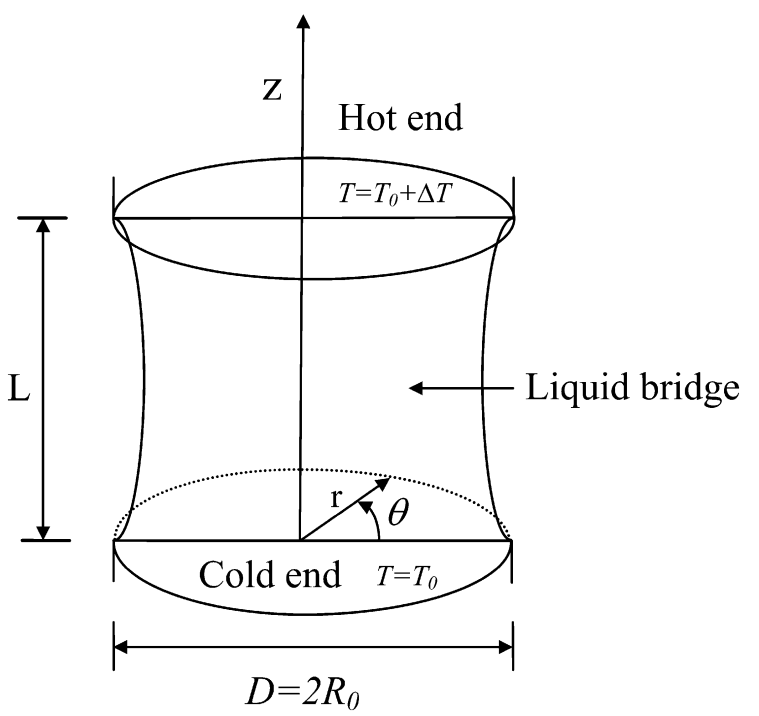

Fig. 1. Schematic of a liquid bridge with rod diameter of $D$ and gap of $L$. temperatures $T_{0}$ and $T_{0}+\Delta T$, respectively. Typical dimensionless parameters such as aspect ratio, volume ratio, Reynolds number, Marangoni number, Prandtl numbers and Biot number are defined as,

$$
\begin{gathered}
A=\frac{L}{2 R_{0}}, \quad V=\frac{\int_{0}^{L} R^{2} \mathrm{~d} z}{R_{0}^{2} L}, \quad R e=\frac{U_{0} R_{0}}{v}, \\
M a=\frac{\left|\sigma_{T}^{\prime}\right| \Delta T R_{0}}{\rho v \alpha}, \quad \operatorname{Pr}=\frac{v}{\alpha}, \quad B i=\frac{h R_{0}}{k}
\end{gathered}
$$

where the reference velocity is $U_{0}=v / R_{0}$, and $R_{0}, \rho, v, \alpha, k$, $h$, and $\sigma_{T}^{\prime}$ denote rod radius, liquid density, kinematic viscosity, thermal diffusivity, thermal conductivity, heat transfer coefficient, and surface tension differentiation with respect to temperature, respectively.

In the linear stability analysis of thermocapillary convection in the floating half-zones, a steady axi-symmetric basic state, $\mathbf{u}_{0}=\left(u_{0}, 0, w_{0}\right), p_{0}$ and $T_{0}$, is obtained through numerical computations. The small amplitude fluctuation of the velocities $\mathbf{u}=(u, v, w)$, pressure $p$ and temperature $T$ are imposed on the basic state. The dimensionless governing equations for the perturbed quantities in the microgravity conditions can be obtained using the Boussinesq approximation as (Chen and $\mathrm{Hu}, 1998$ ),

$$
\begin{aligned}
& \frac{\partial \mathbf{u}}{\partial t}+\mathbf{u} \cdot \nabla \mathbf{u}_{0}+\mathbf{u}_{0} \cdot \nabla \mathbf{u}=-\nabla p+\frac{1}{R e} \nabla^{2} \mathbf{u}, \\
& \nabla \cdot \mathbf{u}=0 \\
& \frac{\partial T}{\partial t}+\mathbf{u} \cdot \nabla T_{0}+\mathbf{u}_{0} \cdot \nabla T=\frac{1}{\operatorname{RePr}} \nabla^{2} T
\end{aligned}
$$

where the length, time and velocity are normalized with $R_{0}$, $R_{0} / U_{0}$ and $U_{0}$, respectively. After applying the curl operation to Eq. (2), we can obtain the vortex equations. In this paper, we used the vortex equations in $r$ and $z$ directions, Eqs. (3) and (4) for solving the eigenvalue problem.

The perturbation quantities $(u, v, w, p, T)$ can be expanded as a sum of the spectral terms, for example,

$$
\left(\begin{array}{l}
u \\
v \\
w \\
p \\
T
\end{array}\right)=\sum_{m} \mathrm{e}^{\sigma t+\operatorname{im} \theta}\left(\begin{array}{l}
\tilde{u}_{m}(r, z) \\
\operatorname{im} \tilde{v}_{m}(r, z) \\
\tilde{w}_{m}(r, z) \\
\tilde{p}_{m}(r, z) \\
\tilde{T}_{m}(r, z)
\end{array}\right)+\text { c.c. }
$$

where $\sigma=\sigma_{r}+\mathrm{i} \sigma_{i}, \sigma_{r}$ and $\sigma_{i}$ are the increasing rate and frequency of small perturbation, respectively, $m$ denotes the azimuthal wave number, $i$ denotes the complex unit $\sqrt{-1}$, and c.c. denotes the complex conjugate. From the above equation, the frequency in Hertz can be obtained as,

$f=\sigma_{i} U_{0} /\left(2 \pi R_{0}\right)=\sigma_{i} \operatorname{Rev} /\left(2 \pi R_{0}^{2}\right)$.

The boundary conditions at the ends $(z=0$ and $z=2 A)$ are given by,

$\mathbf{u}=0, \quad \frac{\partial w}{\partial z}=0, \quad$ and $T=0$,

while the boundary conditions at the free surface are, 


$$
\begin{aligned}
& \mathbf{u} \cdot \mathbf{n}=0, \quad \mathbf{t} \cdot\left(\mathbf{S} \cdot \mathbf{n}+\frac{M a}{\operatorname{RePr}} \nabla T\right)=0, \\
& \mathbf{s} \cdot\left(\mathbf{S} \cdot \mathbf{n}+\frac{M a}{\operatorname{RePr}} \nabla T\right)=0 \quad \text { and } \mathbf{n} \cdot \nabla T=-B i T,
\end{aligned}
$$

where $\mathbf{n}$ denotes the normal unit vector at the free surface, and $\mathbf{t}$ and $\mathbf{s}$ denote the tangential unit vectors in the vertical and horizontal cross-sections, respectively. $\mathbf{S}$ denotes the rate-of-strain tensor.

In the following analysis, an adiabatic condition is assumed at the free surface, which implies $B i=0$. The conditions at the central axis, $r=0$, are taken as,

$u=0, \quad v=0, \quad \frac{\partial w}{\partial r}=0, \quad \frac{\partial T}{\partial r}=0, \quad$ for $m=0$,

$\frac{\partial u}{\partial r}=0, \quad u+\frac{\partial v}{\partial \theta}=0, \quad w=0, \quad T=0, \quad$ for $m=1$,

$u=0, \quad v=0, \quad w=0, \quad T=0, \quad$ for $m>1$.

By substituting the expanded form of the perturbation quantities, Eq. (5), into the perturbation equations, and using the collocation method, the discretized equations for the eigenvalue problem can be obtained (Orszag and Kells, 1980; Orszag and Patera, 1983). It should be noted that the continuity equation boundary conditions are imposed at the rod ends, and the discretized equations for the vortex equation in the $r$ direction at the collocation grid points next to the rods are not included for solving the eigenvalue problem. The eigenvalues and eigenfunctions were obtained by using the Q- $\mathrm{R}$ method. We used $32 \times 32$ Chebyshev polynomials in $r$ and $z$ directions, respectively, and the generalized eigenvalue problems with a complex matrix of $(4 \times 32 \times 32)^{2}$ in size were solved.

\section{Results}

The numerical codes for 2-D basic axisymmetric states and the 3-D linear stability analysis were used to obtain the critical Marangoni numbers for small Prandtl numbers (Chen et al., 1999) and the predicted critical Marangoni numbers for $\operatorname{Pr}=0.01, A=0.5$ and 0.6 , and $V=1$ were used as benchmarks by other researchers (Shevtsova,
2005). We here considered liquid bridges with $P r=100$ and aspect ratio of $A=0.75$. The critical Marangoni numbers and the critical frequencies were obtained for liquid volumes ranging from 0.4 to 1.2 . Table 1 lists the critical Marangoni number, $M a_{\mathrm{c}}$, the critical frequency, $\sigma_{i} R e_{\mathrm{c}}$, and frequency for silicone oil liquid bridges of $60 \mathrm{~mm}$ in rod diameter. As can be seen, the curve of the critical Marangoni number is continuous for $0.4 \leqslant V \leqslant 1.2$. The critical frequencies are not zero meaning that the convection first transits to an oscillatory convection when increasing the Marangoni number. From Eq. (6), it can be seen that the critical frequency is proportional to the inverse of the square of disk radius, meaning that the frequency will decrease when increasing the rod diameter. The frequencies for liquid bridges with disk diameter of $60 \mathrm{~mm}$ and kinetic viscosity of $v=10^{-5} \mathrm{~m}^{2} / \mathrm{s}$ (10 cSt silicone oil) are listed in Table 1. The critical $M a$ is about 15,333 for a liquid bridge with a cylindrical shape, and the minimum critical $M a$ for $0.4 \leqslant V \leqslant 1.2$ is about 8849 occurring at around $V=0.6$.

In the ground experiments, the rod diameter is usually from $2 \mathrm{~mm}$ to $6 \mathrm{~mm}$, and the liquid bridge cannot sustain when the temperature difference is more than $100 \mathrm{~K}$ because of the breakdown of the bridge due to the small surface tension at the high temperature. In the microgravity condition, a large-size liquid bridge can be made, thus the same value of Marangoni number can be realized with relatively small temperature difference.

As observed from the ground-based experiments for $D=2 \mathrm{~mm}$ (Cao et al., 1992) and $D=3 \mathrm{~mm}$ (Hu et al., 1994), there is a gap between the two curves, which corresponds to the region where the critical Marangoni number cannot be obtained on the ground. However, it is possible to obtain a large value of $M a$ number with a large-size liquid bridge in the space experiments. For example, in the D-2 missions on board the German Space laboratory, Albanese et al. (1995) found that the critical Marangoni numbers based on the rod radius are 76,000 for $A=0.75$ and $D=45 \mathrm{~mm}$ and 110,666 for $A=0.75$ and $D=60 \mathrm{~mm}$ while the critical frequencies are $0.021 \mathrm{~Hz}$ and $0.012 \mathrm{~Hz}$. The predicted critical Marangoni numbers by the linear stability analysis are lower

Table 1

The critical Marangoni number and critical frequency for $\operatorname{Pr}=100$ and $A=0.75$, and the frequency in liquid bridges with rod diameter of 60 mm and $v=10^{-5} \mathrm{~m}^{2} / \mathrm{s}(10 \mathrm{cSt}$ silicone oil)

\begin{tabular}{lllll}
\hline Volume $V$ & $\begin{array}{l}\text { The most } \\
\text { unstable mode }\end{array}$ & $\begin{array}{l}\text { Critical } \\
\text { Marangoni number }\end{array}$ & $\begin{array}{l}\text { Critical } \\
\text { frequency } \sigma_{i} R e_{\mathrm{c}}\end{array}$ & $\begin{array}{l}f=\sigma_{i} R e_{\mathrm{c}} v /\left(2 \pi R_{0}^{2}\right)\left(R_{0}=0.03 \mathrm{~m}, v=10^{-5} \mathrm{~m}^{2} / \mathrm{s}\right) \\
\text { (in Hertz) }\end{array}$ \\
\hline 0.4 & $m=1$ & 16,592 & 4.336 & 0.0076 \\
0.5 & $m=1$ & 9600 & 2.739 & 0.0048 \\
0.6 & $m=1$ & 8849 & 2.212 & 0.0039 \\
0.7 & $m=1$ & 17,171 & 2.382 & 0.0042 \\
0.8 & $m=1$ & 16,698 & 2.142 & 0.0038 \\
0.9 & $m=1$ & 15,041 & 2.275 & 0.0040 \\
1.0 & $m=1$ & 15,333 & 1.918 & 0.0034 \\
1.1 & $m=1$ & 17,196 & 2.655 & 0.0047 \\
1.2 & $m=1$ & 10,015 & 0.983 & 0.0017 \\
\hline
\end{tabular}



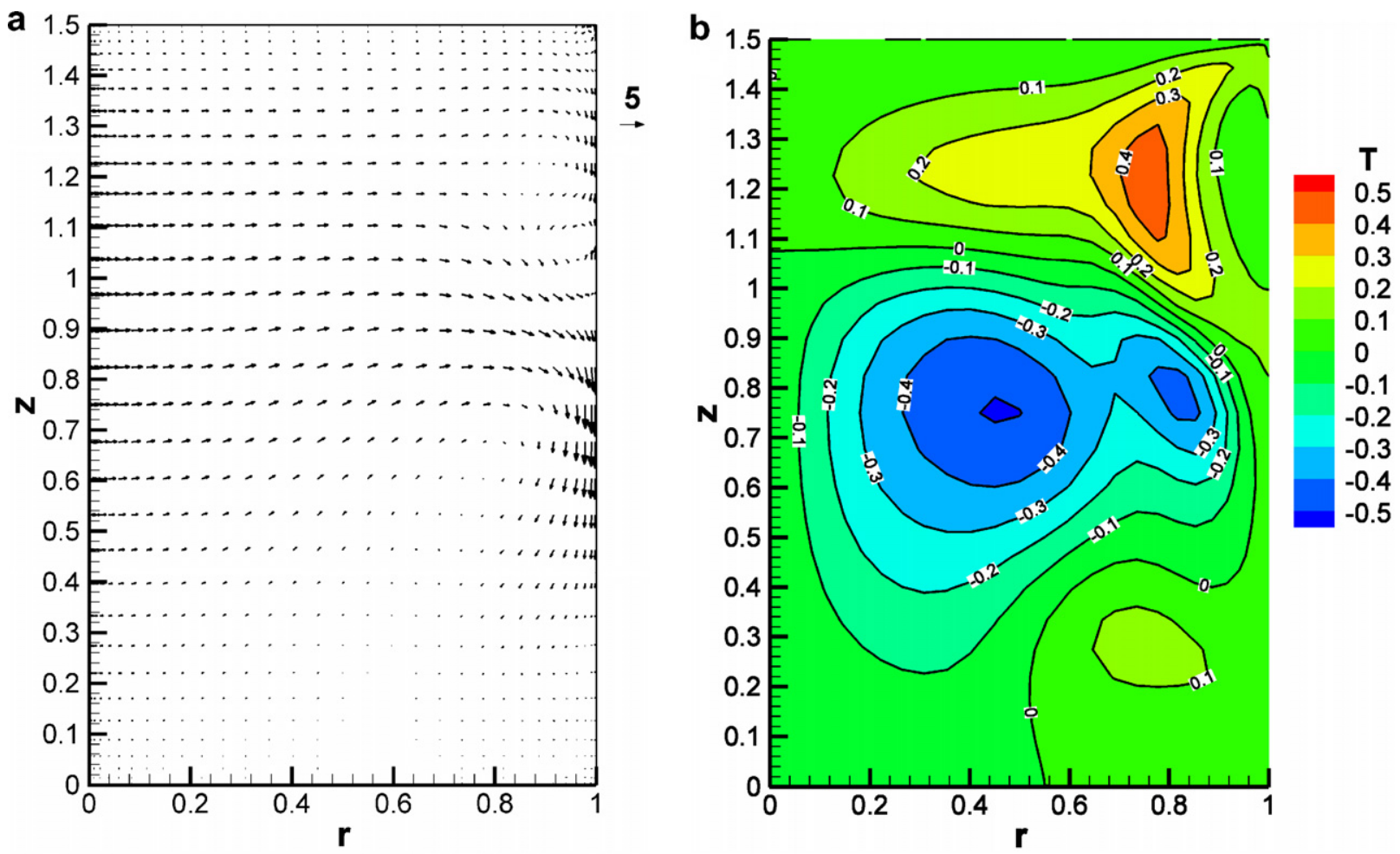

Fig. 2. The flow pattern (a) and temperature distribution (b) of the critical mode $m=1$, when $\operatorname{Pr}=100, A=0.75, V=1$, and $M a=20,000$.

than the values measured in the space experiments (Albanese et al., 1995). The unsteady heating procedures in space experiments caused the differences in the measured critical Marangoni numbers in the space experiments.

The flow and temperature patterns of the critical mode $m=1$ are shown in Fig. 2 for $P r=100, A=0.75, V=1$, and $M a=20,000$. The convection in the liquid bridge is caused by the temperature disturbances on the free surface. There is a vortex rotating clockwise in the right half of the liquid (Fig. 2a) while rotating counter-clockwise in the left half of the liquid. The hot spot on the free surface near the upper rod in Fig. $2 b$ induces a downward flow on the free surface as shown in Fig. 2a. The instability mechanism is of hydrothermal origin for convections in large-Prandtl-number liquid bridges.

\section{Conclusions}

We analyze the critical Marangoni numbers and the critical frequencies by using the linear stability analysis for liquid bridges with $P r=100, B i=0$ and $A=0.75$. It is found that the curve of the critical Marangoni number is continuous for $0.4 \leqslant V \leqslant 1.2$. The most unstable mode is $m=1$ and the critical frequency is not zero meaning that the convection first transits to an oscillatory convection. The critical $M a$ is about 15,333 for a liquid bridge with a cylindrical shape, and the minimum critical $M a$ for $0.4 \leqslant V \leqslant 1.2$ is about 8849 occurring at around $V=0.6$. The predicted critical Marangoni numbers are lower than the values measured in the space experiments since the unsteady heating proce- dures in space experiments caused the differences in the measured critical Marangoni numbers.

\section{Acknowledgements}

This work has been supported by the National Science Foundation of China (10472126, 10432060) and the Knowledge Innovation Program of the Chinese Academy of Sciences.

\section{References}

Albanese, C., Carotenuto, L., Castagnolo, D., Ceglia, E., Monti, R. An investigation on the onset of oscillatory Marangoni flow. Adv. Space Res. 16, 787-794, 1995.

Cao, Z.H., Xie, J.C., Tang, Z.M., Hu, W.R. Experimental study on oscillatory thermocapillary convection. Sci. China 35, 725-733, 1992.

Chen, Q.S., Hu, W.R. Numerical investigation on a simulation model of floating zone convection. Int. J. Heat Mass Transfer 40, 757-763, 1997.

Chen, Q.S., Hu, W.R. Influence of liquid bridge volume on instability of floating half zone convection. Int. J. Heat Mass Transfer 41, 825-837, 1998.

Chen, Q.S., Hu, W.R., Prasad, V. Effect of liquid bridge volume on the instability in small-Prandtl-number half zone. J. Crystal Growth 203, 261-268, 1999.

Hu, W.R., Shu, J.Z., Zhou, R., Tang, Z.M. Influence of liquid bridge volume on the onset of oscillation in floating zone convection. $\mathrm{J}$. Crystal Growth 142, 379-384, 1994.

Nienhüser, C., Kuhlmann, H.C. Stability of thermocapillary flows in noncylindrical liquid bridges. J. Fluid Mech. 458, 35-73, 2002. 
Orszag, S.A., Kells, L.C. Transition to turbulence in plane Poiseuille and plane Couette flow. J. Fluid Mech. 96, 159-205, 1980.

Orszag, S.A., Patera, A.T. Secondary instability of wall-bounded shear flows. J. Fluid Mech. 128, 347-385, 1983.

Shevtsova, V.M., Legros, J.C. Oscillatory convection motion in deformed liquid bridges. Phys. Fluids 10, 1621-1634, 1998.

Shevtsova, V. Thermal convection in liquid bridges with curved free surfaces: Benchmark of numerical solutions. J. Crystal Growth 280, 632-651, 2005.
Sumner, L.B.S., Neitzel, G.P., Fontaine, J.-P., Dell'Aversana, P. Oscillatory thermocapillary convection in liquid bridges with highly deformed free surfaces: experiments and energy-stability analysis. Phys. Fluids 13, 107-120, 2001.

Yao, Y.L., Shu, J.Z., Xie, J.C., Liu, F., Hu, W.R., Hirata, A., Nishizawa, S.I., Sakurai, M. Transition of oscillatory floating half zone convection from earth's gravity to microgravity. Int. J. Heat Mass Transfer 40, 2517-2524, 1997. 